\title{
Influence of dispersing agents on adsorption and desorption rate of a disperse dye on polyester
}

\begin{abstract}
Dispersing agents play important roles in polyester dyeing with disperse dyes. The purpose of this work is to determine the influence of dispersing agents on the adsorption and desorption rate of polyester dyeing with CI Disperse Blue 284. Polyester fabric was dyed at HTHP method using two types of dispersing agents at different concentrations. The performances studied were the changes in dye transfer rate, $\mathrm{K} / \mathrm{S}$ values of the dyed samples and dye bath concentration. The rate of adsorption and desorption of disperse dyes were affected significantly not only by the type but also the concentration of dispersing agents.
\end{abstract}

Keywords: disperse dyes, dispersing agents, adsorption and desorption rate, polyester dyeing

\author{
Volume 6 Issue 5 - 2020 \\ Md Koushic Uddin,' Sonia Hossain,' Umma \\ Habiba, ${ }^{2}$ Md Nafiur Rahman, ${ }^{2}$ Rayhan \\ Mahmud $^{2}$ \\ 'Assistant Professor, Department of Textile Engineering, \\ Ahsanullah University of Science \& Technology, Bangladesh \\ ${ }^{2}$ Graduating student, Department of Textile Engineering, \\ Ahsanullah University of Science \& Technology, Bangladesh
}

Correspondence: Md. Koushic Uddin, Assistant Professor, Ahsanullah University of Science and Technology, Dhaka, Bangladesh,Tel 01818252577,Email koushic_tex@aust.edu

Received: September 30, 2020 | Published: October 05, 2020

\section{Introduction}

Polyester fibre generally consisting of linear chain polymers containing ester groups ${ }^{1}$ and is derived from dicarboxylic acids and diols. ${ }^{2}$ It is the highest volume synthetic fiber produced globally due to its unique end use and easy care properties. ${ }^{3,4}$ The polyester fibres are highly crystalline and are usually even and rod like shapes. ${ }^{5}$ Owing to their hydrophobic nature they are therefore difficult to dye at low temperatures and dyeing is usually carried out at high temperatures, regularly around $130^{\circ} \mathrm{C} \cdot 6,7$

Disperse dyes usually used to dye polyester fibres which are non-ionic in nature and have limited water solubility even at high dyeing temperatures. ${ }^{8}$ Therefore, require the presence of a dispersing agent, which improves their solubility. ${ }^{9}$ These agents produce energy barriers maintaining dispersions in their meta-stable condition, thus, avoiding the aggregation of dispersed particles thus improves the dispersion stability, solubility, leveling, and fastness properties. ${ }^{10}$ Since the dispersion of disperse dyes is thermodynamically unstable, the particle sizes tend to increase due to the decreased free energy of the system. ${ }^{11}$ The stability of the dye dispersion and the rate of dyeing are all affected by the type and concentration of the dispersing agents. ${ }^{8}$

So the incorporation of dispersing agent which usually a surfactant in the dyebath is a very important factor in the application of disperse dyes. The hydrophobic tails inside the micelle of the surfactant molecules which actually reduce the particle size and solubilize the disperse dye molecules. Then soluble dyes as soon as transferred to the fibre free the micelles results they re-from and dissolve more dyes from the dye reservoir. ${ }^{12}$

The stabilization of the dispersion is based on several effects depending on the dispersion agent used. It may be either an electrostatic stabilization, where the charge of the dispersing agent plays an essential part, or a steric stabilization, where the chemical construction and the molecular weight of the dispersing agent are the key factors. ${ }^{13}$

Polyester fibers dyeing with disperse dyes is a complex process and the rate of dye uptake, solubility and the dispersion of the dye in the dye bath are depends on the different auxiliaries used in the bath. ${ }^{14,15}$ The aim of this study is to analyze the dependency of dispersing agents on the adsorption and desorption of disperse dyes on the dyeing of polyester fabric.

\section{Experimental}

\section{Material}

Factory washed double jersey $100 \%$ polyester knitted fabric $(250 \mathrm{~g} /$ $\mathrm{m}^{2}$ ) was used. A commercial disperse dye sample of CI Disperse Blue 284 was used without further processing. Anionic and nonionic type dispersing agents were used and described in the Table 1.

Table I Specifications of dispersing agents used in this study

\begin{tabular}{lll}
\hline Agent No. & Type & Chemical Nature \\
\hline I & Anionic & $\begin{array}{l}\text { Mixture of aromatic sulphonates and } \\
\text { carboxylic acid polyglycol ester }\end{array}$ \\
2 & Nonionic & Product of APEO free ethoxylates \\
\hline
\end{tabular}

\section{Dyeing}

Before dyeing polyester fabric was washed with acetic acid at $60^{\circ} \mathrm{C}$ for 10 minutes to remove impurities. Dyeing was carried out for $3 \%$ shade in Data colour Ahiba IR sample dyeing machine at $130^{\circ} \mathrm{C}$ for 70 minutes. Three different concentrations of each dispersing agent were selected accordingly from the provided manufacturing brochure. For anionic and nonionic dispersing agent the concentrations used were $1 / 2 / 3 \mathrm{~g} / \mathrm{L}$ and $0.25 / 0.5 / 0.75 \mathrm{~g} / \mathrm{L}$ respectively.

\section{Adsorption/desorption rate and colour strength}

The rates of adsorption and desorption were calculated from the relevant absorbance curves from the dyed liquor after 6 (six) predetermined time intervals at $130^{\circ} \mathrm{C}$. Colour strength values $(\mathrm{K} / \mathrm{S})$ of the dyed samples were measured using the Kubelka-Munk equation of the same time intervals. Data Colour Dual-Beam Spectrophotometer 650 was used. For accuracy 6 (six) different dyeing baths were set 
maintaining exactly the same configuration. At each time interval one bath was taken out for assessment. $1^{\text {st }}$ bath was assessed at 1 minute after reaching $130^{\circ} \mathrm{C}$ consequently $2^{\text {nd }}, 3^{\text {rd }}, 4^{\text {th }}, 5^{\text {th }}$ and $6^{\text {th }}$ bath were assessed at 15, 30, 45, 60 and 70 minutes respectively.

\section{Results and discussion}

According to Beer-Lamberts law the absorbance of a solution at a given wavelength is directly proportional to its concentration. Table 2 and Figure 1 presents the absorbance/concentration values and curve of the disperse dye used in this work. The attenuation co-efficient of the dye at $\lambda_{540} \mathrm{~nm}$ was $3 \times 10^{-3} \mathrm{Lg}^{-1} \mathrm{~cm}^{-1}$.

Table 2 Absorbance Values at different concentration

\begin{tabular}{lll}
\hline Sample & Concentration $(\mathbf{m g} / \mathbf{L})$ & Absorbance \\
\hline 1 & 25 & 0.268 \\
2 & 30 & 0.2876 \\
3 & 50 & 0.351 \\
4 & 100 & 0.535 \\
5 & 150 & 0.7121 \\
6 & 200 & 0.9179
\end{tabular}

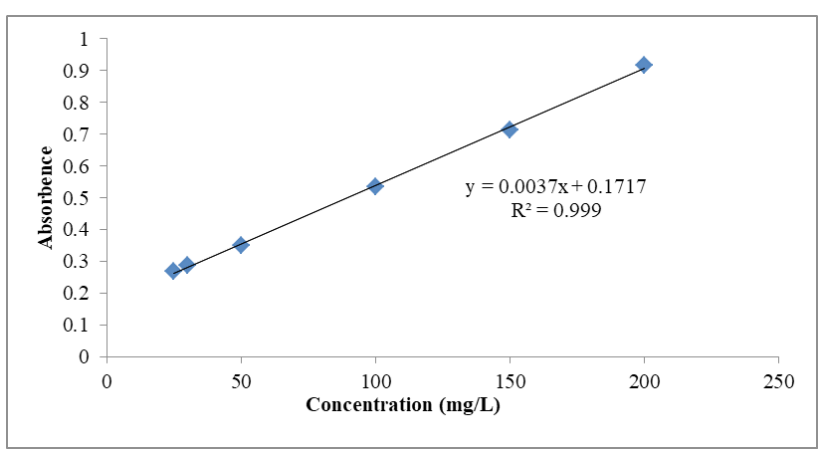

Figure I Absorbance vs concentration curve.

Figure 2 and Figure 3 presents the adsorption and desorption rate at $130^{\circ} \mathrm{C}$ of used disperse dyes on polyester influenced by different dispersing agent. The initial adsorption rate for anionic dispersing agent (AD) was found almost 30 to $40 \%$ where as in case of nonionic dispersing agent (ND) it was quite high and almost 55 to $60 \%$.

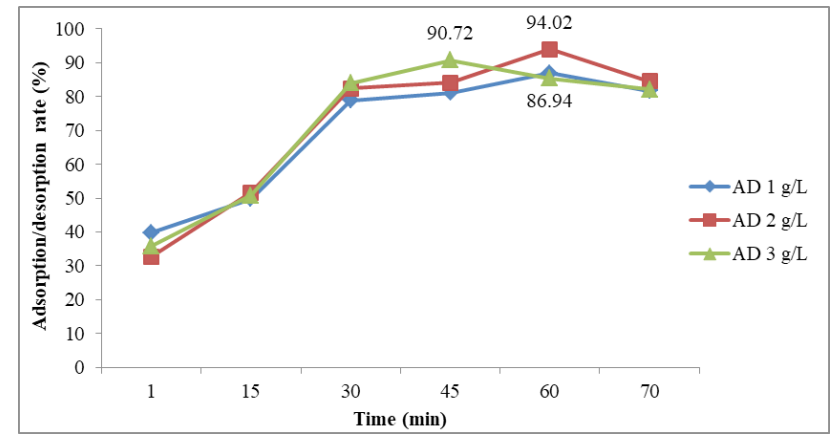

Figure 2 Adsorption/desorption rate (\%) influenced by anionic dispersing agent (AD).

In case of anionic dispersing agent the maximum adsorption achieved after 60 minutes of dyeing at $130^{\circ} \mathrm{C}$ for 1 and $2 \mathrm{~g} / \mathrm{L}$ but for $3 \mathrm{~g} / \mathrm{L}$ the maximum adsorption achieved after 45 minutes of dyeing.
Same trend observed in case of nonionic dispersing agent. Maximum adsorption achieved after 30 minutes of dyeing at $130^{\circ} \mathrm{C}$ for higher concentration. It's clear that in case of nonionic dispersing agent the adsorption rate is higher that achieved in case of anionic dispersing agent. At high concentration of dispersing agents took less dyeing time to achieve the maximum adsorption.

Maximum adsorption found $94.02 \%$ for $2 \mathrm{~g} / \mathrm{L}$ concentration of anionic dispersing agent at 60 minutes of dyeing where as $92.88 \%$ found for $0.5 \mathrm{~g} / \mathrm{L}$ concentration of nonionic dispersing agent at 45 minutes of dyeing. It can be concluded that the adsorption rate is quite high when used nonionic dispersing agent compare to anionic dispersing agent.

Figure 2 and Figure 3 also presents the desorption rate of the dyes. The desorption rate of anionic dispersing agent is higher than nonionic dispersing agent. After 70 minutes of dyeing around 10$15 \%$ desorption of dyes occurred when anionic dispersing agent was used which is quite higher than nonionic dispersing agent and also desorption occurred at slower rate.

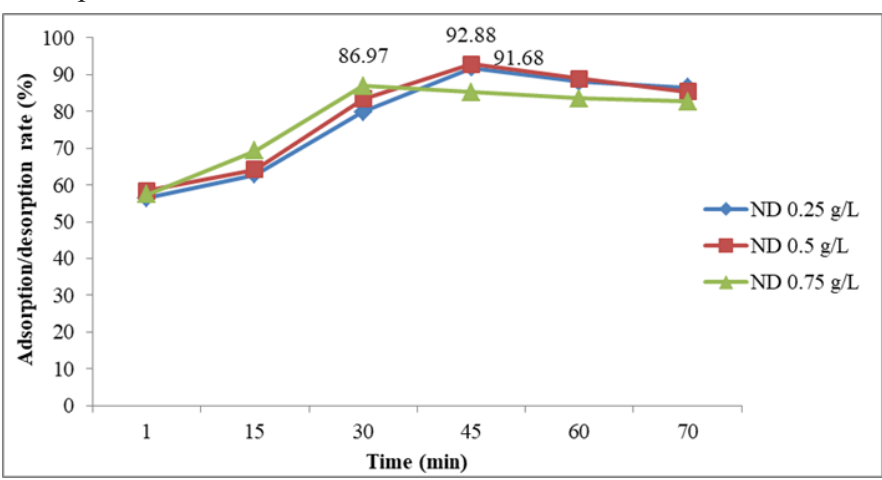

Figure 3 Adsorption/desorption rate (\%) influenced by nonionic dispersing agent (ND).

At different concentration of dispersing agent the adsorption rate is similar but at higher concentration dye desorption occurred little faster. In both cases medium concentration showed good and stable results.

Figure 4 and Figure 5 presents the colour strength values of the dyed samples at time intervals. Maximum colour strength achieved after 45-60 minutes of dyeing. After 60 minutes of dyeing colour strength of dyed samples started to fall. In case of anionic dispersing agent the the initial dye pick up rate is low and desorption rate also high after 60 minutes of dyeing.

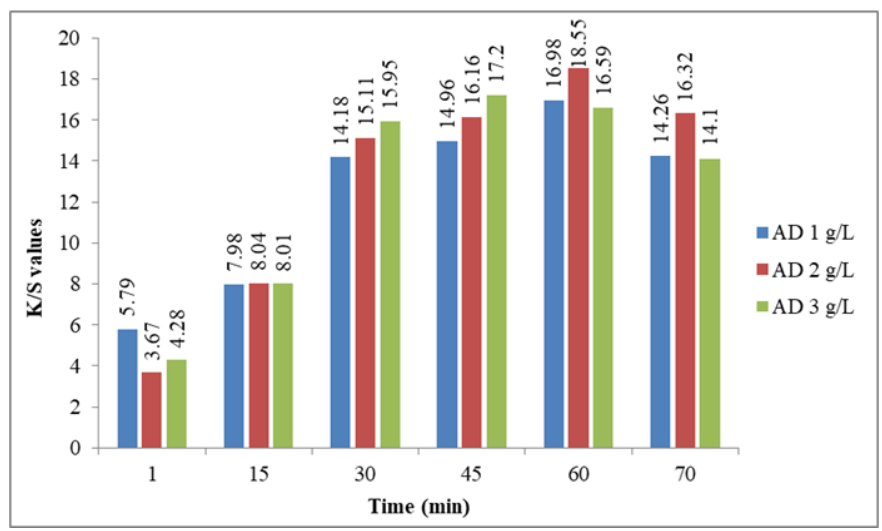

Figure 4 Colour yield values influenced by anionic dispersing agent (AD). 


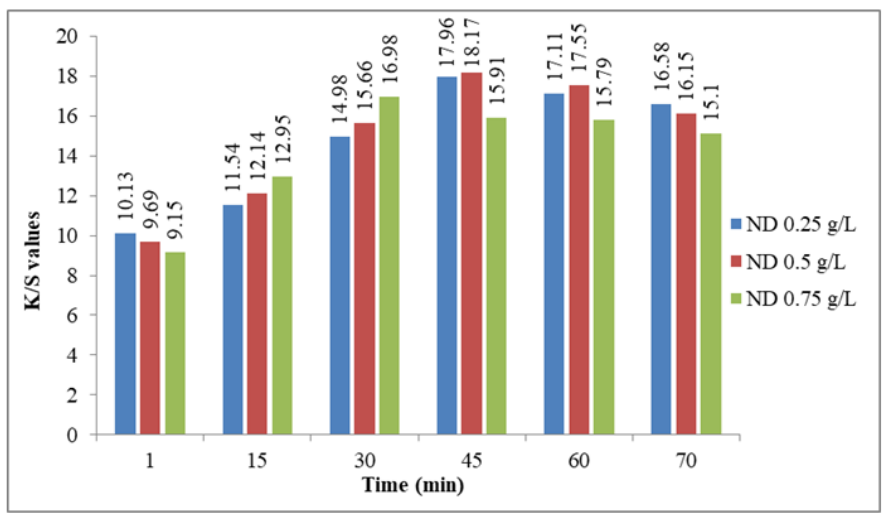

Figure 5 Colour yield values influenced by nonionic dispersing agent (ND).

The initial dye pick up is quite high for nonionic dispersing agent and the desorption rate is also low after 60 minutes of dyeing.

\section{Conclusion}

This work clearly shows that different types of dispersing agents have different adsorption and desorption rates on polyester dyeing with disperse dyes. The dyeing process is quite complex and the dispersing agents plays an important role which can't be ignored. Not only the type but also the concentration of dispersing agents can affect the rate of adsorption and desorption of disperse dyes. The proper selection of the type of dispersing agent and their concentration are very crucial for efficient and successful dyeing.

\section{Acknowledgments}

None.

\section{Funding}

None.

\section{Conflicts of interest}

The authors have no conflicts of interest regarding the publication of this paper.

\section{References}

1. McIntyre JE. Synthetic fibres: nylon, polyester, acrylic, polyolefin. US: Taylor \& Francis; 2005.

2. Joshi M. The impact of nanotechnology on polyesters, polyamides and other textiles. Polyesters and Polyamides. 2008:354-415.

3. Perepelkin K. Polyester Fibres. Fibre Chemistry. 2001;33(5):333-339.

4. Menachem L. Hand book of fibre chemistry. 3rd edn. Taylor \& Francis ebooks; 2006.

5. Needles HL. Textile fibers, dyes, finishes, and processes: a concise guide. 1986.

6. Hawkyard C. Synthetic fibre dyeing. Society of Dyers and Colourists; 2004.

7. Park J, Shore J. Practical dyeing Volume 2-Fibre types and dyeing processes. Society of Dyers and Colourists; 2004. 47 p.

8. Odvarka J, Schejbalova $\mathrm{H}$. The effect of dispersing agents on the dyeing of polyester with a disperse dye. Journal of the Society of Dyers and Colourists. 1994;110(1):30-34.

9. Al-Etaibi AM, Alnassar HS, El-Apasery MA. Dyeing of polyester with disperse dyes: Part 2. Synthesis and dyeing characteristics of some azo disperse dyes for polyester fabrics. Molecules. 2016;21(7):855.

10. Gharanjig K, Dadras F Sadr, Sadeghi-Kiakhani M, et al. Stability of dye dispersions in the presence of various surface active agents and additives. Taylor \& Francis Online. 2012:381-388.

11. Chung YS, Son EJ, Lee KW. Dispersion behavior of CI Disperse Yellow 54 in high temperature dyeing. Textile Research Journal. 2001;71(2):174-178.

12. Johnson A. The theory of coloration of textiles. Bradford: Society of Dyers and Colourists; 1989.

13. U1 Aleem A. An investigation of alternatives to reductive clearing in the dyeing of polyester. Doctoral dissertation, Heriot-Watt University; 2013.

14. Al-Etaibi A, El-Apasery MA, Al-Awadi N. The effect of dispersing agent on the dyeing of polyester fabrics with disperse dyes derived from 1, 4-diethyl-2, 6-dioxo-1, 2, 5, 6-tetrahydropyridine-3-carbonitrile. European Journal of Chemistry. 2013;4(3):240-244.

15. Kabir SM, Koh J. Effect of chelating agent in disperse dye dyeing on polyester fabric. Fibers and Polymers. 2017;18(12):2315-2321. 